\title{
Associations between vitamin D status and radial bone geometry in older South Asian and Caucasian women
}

\author{
A. L. Darling ${ }^{1}$, O. A. Hakim ${ }^{1}$, K. Horton $^{2}$, M. A. Gibbs ${ }^{2}$, L. Cui $^{3}$, J. L. Berry ${ }^{4}$, S. A. Lanham-New ${ }^{1}$ \\ and K. H. Hart ${ }^{1}$ \\ ${ }^{1}$ Department of Nutrition and Metabolism, Faculty of Health and Medical Sciences, University of Surrey, Guildford, \\ ${ }^{2}$ School of Health and Social Care, Faculty of Health and Medical Sciences, University of Surrey, Guildford, ${ }^{3}$ Faculty of \\ Engineering and Physical Sciences, University of Surrey, Guildford and ${ }^{4}$ University of Manchester, Manchester, UK
}

It is unknown whether there is an association between vitamin D status and bone geometry parameters in older South Asian women, and whether this differs from same-age Caucasian women. This is the first study, to the authors' knowledge, to assess the relationship between vitamin D status and peripheral quantitative computed tomography (pQCT) indices of bone architecture in older South Asian women. To measure radius bone structure, pQCT scans (Stratec X2000L) at the distal (4\%) and mid-shaft $(66 \%)$ sites were undertaken in $n=18$ South Asian (mean age $63.5 \mathrm{y}+/-3.6$ ) and $n=50$ Caucasian women (mean age $65.9 \mathrm{y}+/-4.8$ ). A fasting blood sample was obtained for assessment of vitamin D status (25-hydroxyvitamin D). Partial correlations assessed the relationship between vitamin D status and the radial bone indices. Unadjusted and BMI adjusted data can be seen in the table.

\begin{tabular}{|c|c|c|c|c|c|c|c|c|c|}
\hline & \multirow[b]{3}{*}{ Parameter/Site } & \multicolumn{4}{|c|}{ Caucasian $n=50$} & \multicolumn{4}{|c|}{ South Asian $n=18$} \\
\hline & & \multicolumn{2}{|c|}{ Unadjusted } & \multicolumn{2}{|c|}{ BMI } & \multicolumn{2}{|c|}{ Unadjusted } & \multicolumn{2}{|c|}{ BMI } \\
\hline & & $r$ & $p$ & $r$ & $p$ & $r$ & $p$ & $r$ & $p$ \\
\hline Mass & $\begin{array}{l}\text { Mass } \mathrm{g} / \mathrm{cm}^{3} 4 \% \\
\text { Mass } \mathrm{g} / \mathrm{cm}^{3} 66 \%\end{array}$ & $\begin{array}{l}0.21 \\
0.17\end{array}$ & $\begin{array}{l}0.16 \\
0.27\end{array}$ & $\begin{array}{l}0.40 \\
0.38\end{array}$ & $\begin{array}{l}0.01 \\
0.01\end{array}$ & $\begin{array}{l}0.26 \\
0.14\end{array}$ & $\begin{array}{l}0.31 \\
0.60\end{array}$ & $\begin{array}{l}0.23 \\
0.10\end{array}$ & $\begin{array}{l}0.41 \\
0.73\end{array}$ \\
\hline Area & $\begin{array}{l}\text { Total Area } \mathrm{mm}^{2} 4 \% \\
\text { Total Area } \mathrm{mm}^{2} 66 \% \\
\text { Trabecular Area } 4 \% \\
\text { Cortical Area } 66 \%\end{array}$ & $\begin{array}{l}0.22 \\
0.11 \\
0.22 \\
0.21\end{array}$ & $\begin{array}{l}0.14 \\
0.48 \\
0.14 \\
0.17\end{array}$ & $\begin{array}{l}0.33 \\
0.25 \\
0.33 \\
0.34\end{array}$ & $\begin{array}{l}0.04 \\
0.12 \\
0.03 \\
0.03\end{array}$ & $\begin{array}{l}0.03 \\
0.13 \\
0.03 \\
0.21\end{array}$ & $\begin{array}{l}0.91 \\
0.63 \\
0.91 \\
0.42\end{array}$ & $\begin{array}{r}-0.03 \\
0.12 \\
-0.03 \\
0.18\end{array}$ & $\begin{array}{l}0.93 \\
0.66 \\
0.93 \\
0.52\end{array}$ \\
\hline Density & $\begin{array}{l}\text { Total Density } \mathrm{mg} / \mathrm{cm}^{3} 4 \% \\
\text { Total Density } \mathrm{mg} / \mathrm{cm}^{3} 66 \% \\
\text { Trab. Density } \mathrm{mg} / \mathrm{cm}^{3} 4 \% \\
\text { CorticalDensitymg/cm } 66 \%\end{array}$ & $\begin{array}{r}-0.02 \\
0.07 \\
-0.07 \\
0.20\end{array}$ & $\begin{array}{l}0.92 \\
0.67 \\
0.66 \\
0.19\end{array}$ & $\begin{array}{l}0.09 \\
0.07 \\
0.03 \\
0.18\end{array}$ & $\begin{array}{l}0.55 \\
0.64 \\
0.85 \\
0.25\end{array}$ & $\begin{array}{r}0.39 \\
-0.04 \\
0.54 \\
0.05\end{array}$ & $\begin{array}{l}0.12 \\
0.88 \\
0.02 \\
0.84\end{array}$ & $\begin{array}{r}0.37 \\
-0.11 \\
0.55 \\
0.02\end{array}$ & $\begin{array}{l}0.17 \\
0.70 \\
0.04 \\
0.96\end{array}$ \\
\hline Strength & $\begin{array}{l}\text { SSIpol } \mathrm{mm}^{3} 66 \% \neq \\
\text { Fracture Load (N) } 66 \% \neq \neq\end{array}$ & $\begin{array}{l}0.19 \\
0.20\end{array}$ & $\begin{array}{l}0.23 \\
0.20\end{array}$ & $\begin{array}{l}0.29 \\
0.34\end{array}$ & $\begin{array}{l}0.07 \\
0.03\end{array}$ & $\begin{array}{l}0.32 \\
0.13\end{array}$ & $\begin{array}{l}0.21 \\
0.61\end{array}$ & $\begin{array}{l}0.33 \\
0.10\end{array}$ & \\
\hline
\end{tabular}

$\neq$ SSIpol $=$ polar strength strain index (predicted measure of ability to resist torsion forces); $\neq \neq \neq$ Fracture Load = predicted amount of Newtons required to fracture the bone

Unadjusted = unadjusted data; BMI = BMI (Body Mass Index) adjusted data.

For BMI adjusted data, in Caucasians, there were significant correlations between vitamin D status and bone mass $(r=0.379 p=0.013)$, strength strain index $(r=0.337 p=0.029)$, area of the cortical layer $(r=0.343 p=0.026)$ and predicted fracture load $(r=0.337$ $p=0.029)$ at the mid-shaft $(66 \%)$ site. There were also positive correlations between vitamin D status and total area $(r=0.327 p=0.035)$ at the distal (4\%) site. Therefore, in Caucasians, increased vitamin D was associated with increased bone mass, size and measures of bone strength at both distal and mid shaft sites. For Asians, the only significant correlations between vitamin D status and the bone parameters were for trabecular density $(r=0.547 p=0.035)$ at the distal $(4 \%)$ site, and there were no significant correlations at the mid shaft $(66 \%)$ site. This suggests, in Asians, increased vitamin D status was only associated with increased density in the trabecular layer, and only at the distal site (4\%). Overall, vitamin D appears to be positively correlated with size, strength and mass parameters in Caucasians, and positively correlated with trabecular density in South Asians. Therefore, in both ethnic groups, vitamin D is associated with positive effects on radial bone parameters, and a stronger bone structure. However, the underlying mechanisms in the two ethnic groups may be different. Further research is required to investigate these differences. 